2013

\title{
Voltage-Controlled Enzyme-Catalyzed Glucose-Gluconolactone Conversion Using A Field-Effect Enzymatic Detector
}

\author{
Siu Tung Yau \\ Cleveland State University, s.yau@csuohio.edu \\ Yan Xu \\ Cleveland State University, y.xu@csuohio.edu \\ Yang Song \\ Cleveland State University \\ Ye Feng \\ Cleveland State University \\ Jiapeng Wang \\ Cleveland State University
}

Follow this and additional works at: https://engagedscholarship.csuohio.edu/scichem_facpub

Part of the Biochemistry Commons, and the Chemistry Commons

How does access to this work benefit you? Let us know!

\section{Recommended Citation}

Yau, Siu Tung; Xu, Yan; Song, Yang; Feng, Ye; and Wang, Jiapeng, "Voltage-Controlled Enzyme-Catalyzed Glucose-Gluconolactone Conversion Using A Field-Effect Enzymatic Detector" (2013). Chemistry Faculty Publications. 225.

https://engagedscholarship.csuohio.edu/scichem_facpub/225

This Article is brought to you for free and open access by the Chemistry Department at EngagedScholarship@CSU. It has been accepted for inclusion in Chemistry Faculty Publications by an authorized administrator of EngagedScholarship@CSU. For more information, please contact library.es@csuohio.edu. 


\title{
Voltage-controlled enzyme-catalyzed glucose- gluconolactone conversion using a field-effect enzymatic detector ${ }^{\dagger}$
}

\author{
Siu-Tung Yau, Yan Xu, Yang Song, Ye Feng and Jiapeng Wang
}

The field effect enzymatic detection (FEED) technique was used to control the kinetics of the enzymatic conversion of glucose to gluconolactone. The glucose gluconolactone conversion occurring at an enzyme immobilized electrode, a well studied process, was confirmed using mass spectrometry. Electro chemical studies showed that the glucose oxidation current depends on the gating voltage $V_{\mathrm{G}}$ and the ion concentration of the sample solution. Additionally, the depletion of glucose in the sample also showed a dependence on $V_{G}$. FEED was used to detect $\mathrm{H}_{2} \mathrm{O}_{2}$ on the zepto molar level in order to show the ultrasensitive detection capability of the technique. These results, while providing evidence for the proposed mechanism of FEED, indicate that $V_{G}$ controls the conversion process. The effect of $V_{G}$ on the glucose gluconolactone conversion was demonstrated by the observed $V_{\mathrm{G}}$ dependent kinetic parameters of the conversion process.

\section{Introduction}

Controlling the kinetics of enzymatic reactions by way of engineered electron transfer ${ }^{1,2}$ at the interface between redox enzymes and electrodes has important implications in biosensors, biofuel cells, and biochemical syntheses. ${ }^{3-5}$ When organisms are used in these applications, the breakdown of glucose is an important energy generation process via complicated enzyme pathways. For instance, it is desirable to convert sugars derived from biomass directly to electricity using microbial fuel cells. ${ }^{3}$ Also, glucose is an essential reactant in the synthesis of long-chain fuels in Escherichia coli. ${ }^{4}$ Enhanced interfacial electron transfer has been obtained by modifying the electrode surface with nanoparticles ${ }^{6}$ or engineering of enzymes/proteins. ${ }^{7}$

Recently, an electrochemical enzymatic detection technique has been developed for ultrasensitive detection. ${ }^{8}$ The field-effect enzymatic detection (FEED) technique, which incorporates an external gating voltage in the conventional electrochemical cell, has provided detection limits for glucose in the zepto molar range ${ }^{9}$ due to the intrinsic current amplification of the detection system. The amplification was believed to be caused by an ioninduced electric field at the electrode-enzyme-solution interface as the result of applying a gating voltage to the working electrode. The field reduced the tunnel barrier between the active site of the enzyme and the electrode. ${ }^{10,11}$ In addition to being a detection method, FEED has been used to control the reduction of $\mathrm{H}_{2} \mathrm{O}_{2}$ occurring at an enzyme-immobilized electrode. ${ }^{12}$

In the present work, we present a detailed study on the voltagecontrolled enzymatic catalysis of the glucose-gluconolactone conversion using the FEED technique. The results of our electrochemical measurements, while providing evidence for the mechanism of FEED, show insights into the kinetics of the conversion. We show that the conversion kinetics could be manipulated using a gating voltage. Since glucose-gluconolactone conversion in organisms is a degradative process, ${ }^{13}$ i.e. the hexose monophosphate pathway via dehydrogenase and the pathway in Aspergillus, Pseudomonas and Gluconobacter spp. catalyzed by glucose oxidase, the present work shows the feasibility of controlling the kinetics of cellular glucose metabolic processes at the surface of an electrode.

\section{The FEED system}

The detection system has been demonstrated previously. ${ }^{8}$ A detailed description of the detection system and its principle of operation are given in the ESI $\dagger$ to provide background information for the reader. Briefly, the system consists of a conventional three-electrode electrochemical cell modified with additional gating electrodes for applying a gating voltage $V_{\mathrm{G}}$ to the working electrode, upon which glucose oxidase (GOx), a redox enzyme, is immobilized (see Fig. S1(a), ESI $\dagger$ ). $V_{\mathrm{G}}$ induces charges at the electrode-solution interface to set up electric 
fields about GOx. Electrons tunnel between the active site of GOx and the electrode. The electron transfer rate constant $k_{\text {et }}$ for the electrode-active site system is $k_{\text {et }} \propto \exp (\beta d),{ }^{14}$ where $d$ is the distance between the electrode and the active site and $\beta$ is the attenuation coefficient, which is proportional to the square root of the tunnel barrier height $\left(\beta \propto\left(\Phi_{\mathrm{B}}\right)^{1 / 2}\right)$. The induced electric field reduces the effective height of the tunnel barrier ${ }^{15,16}$ and results in a smaller value of $\beta$. Therefore $k_{\text {et }}$ and hence the current is enhanced, ${ }^{11,17}$ resulting in amplified signal current.

\section{Experimental}

The working electrode used in this work was made by adsorbing GOx (Sigma Aldrich) or microperoxidase-11 (MP-11) (Sigma Aldrich) to a bare pyrolytic graphite (PG) electrode within a $0.5 \mathrm{~mm} \times 0.5 \mathrm{~mm}$ area defined using a mask. To form a molecule-immobilized electrode, a $0.1 \mathrm{~mL}$ drop of the GOx or MP-11 solution was deposited on the PG electrode and the electrode was then incubated at room temperature for 4 hours. The phosphate buffer saline (PBS) used in this work was prepared using de-ionized water $(18.2 \Omega \mathrm{cm})$. A piece of $0.5 \mathrm{~mm}$-diameter copper wire coated with a thin layer of insulator (enamel) was used as the gating electrode. The wire was bent to form a U-shaped structure, which was attached to the working electrode next to the immobilized enzyme molecules. The electrochemical cell was driven by a commercial electrochemical controller (CH Instruments 660C). A commercial Ag/AgCl (3 M KCl) electrode was used as the reference electrode and a platinum wire was used as the counter electrode. A potential scan rate of $30 \mathrm{mV} \mathrm{s}^{-1}$ was used in recording cyclic voltammograms (CV). Research grade glucose, the C-13 labeled stable heavy isotope of glucose (D-glucose- ${ }^{13} \mathrm{C}_{16}$ ), and hydrogen peroxide $\left(\mathrm{H}_{2} \mathrm{O}_{2}\right)$ were purchased from Sigma Aldrich and used as received. All CV measurements presented here were made using deaerated PBS at room temperature.

Electrospray ionization mass spectrometry (ESI-MS) of the glucose samples was performed using an Applied Biosystems (Foster City, CA) Q-Star Elite quadrupole-time-of-flight mass spectrometer equipped with a TurboIon spray source mode. Samples were diluted by adding the same volumes of $90 \%$ acetonitrile and $10 \%$ ammonium acetate $(200 \mathrm{mM}, \mathrm{pH} 9.0)$. The diluted samples were infused at a rate of $10 \mu \mathrm{L} \mathrm{min}{ }^{-1}$, and data were collected for $0.5 \mathrm{~min}$. Spectra were analyzed using the Applied Biosystems Analyst program.

BREEZE ${ }^{\mathbb{R}} 2$ blood glucose test strips and a BREEZE ${ }^{\mathbb{R}} 2$ blood glucosemeter (Bayer Health Care, Mishawaka, WI) were used to measure the concentration of glucose in the samples. Commercial blood glucose sensors provide a wide measuring range of $20-600 \mathrm{mg} \mathrm{dL} \mathrm{dL}^{-1}(1.11-33.33 \mathrm{mM})$. The concentration of the glucose samples was adjusted to be within this range for accurate measurements.

\section{Results and discussion}

\section{Mass spectrometry}

The GOx-catalyzed conversion of glucose to gluconolactone at electrodes has been well studied. This process was confirmed

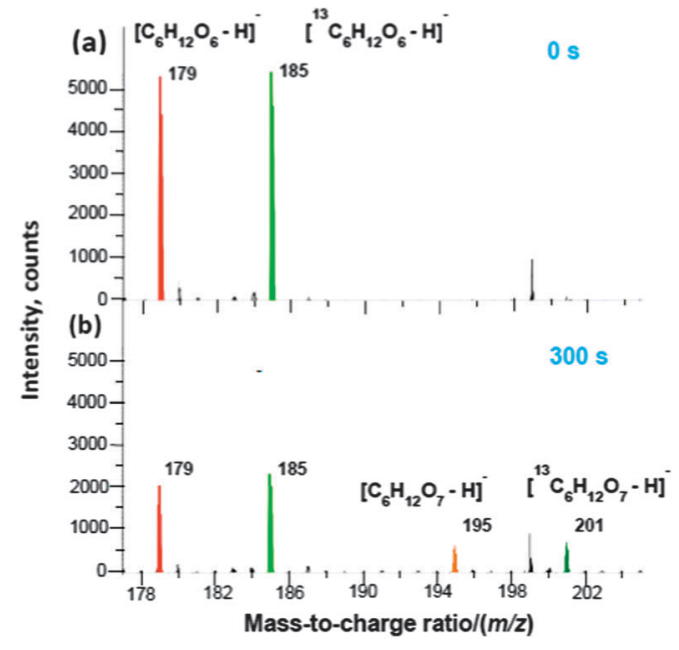

Fig. 1 Mass spectra showing the glucose gluconolactone conversion. (a) A spectrum showing glucose and ${ }^{13} \mathrm{C}_{6}$ glucose at $t=0 \mathrm{~s}$. (b) The decrease in glucose and ${ }^{13} \mathrm{C}_{6}$ glucose and the evolution of gluconolactone and the enzymatic reaction product of ${ }^{13} \mathrm{C}_{6}$ alucose at $t=300 \mathrm{~s}$

using mass spectroscopy in order to provide credibility for the present work. The samples were prepared by performing chronoamperometry with solutions that contained equal amounts of glucose and its C-13 labeled stable heavy isotope, using a GOximmobilized electrode polarized at $0.7 \mathrm{~V} v$ s. $\mathrm{Ag} / \mathrm{AgCl}$. The use of the isotope provided an internal tracer for the enzyme-catalyzed oxidation reaction. Fig. 1 shows the mass spectra of a glucose sample, prepared at two different times of chronoamperometry. Fig. 1(a) shows the spectrum of the sample solution prior to chronoamperometry. It shows that the mass-to-charge ratios $(\mathrm{m} / \mathrm{z})$ of deprotonated glucose $\left[\mathrm{C}_{6} \mathrm{H}_{12} \mathrm{O}_{6}-\mathrm{H}\right]^{-}$and deprotonated stable heavy isotope $\left[{ }^{13} \mathrm{C}_{6} \mathrm{H}_{12} \mathrm{O}_{6}-\mathrm{H}\right]^{-}$are at 179 and 185 , respectively. The spectrum in Fig. 1(b) was obtained with the same sample after $300 \mathrm{~s}$ of chronoamperometry, showing the presence of gluconic acid (gluconolactone dissolved in aqueous solution) $\left[\mathrm{C}_{6} \mathrm{H}_{12} \mathrm{O}_{7}-\mathrm{H}\right]^{-}$and the acid due to the stable heavy isotope $\left[{ }^{13} \mathrm{C}_{6} \mathrm{H}_{12} \mathrm{O}_{7}-\mathrm{H}\right]^{-}$at $m / z 195$ and $m / z$ 201, respectively. Fig. 1 shows that while the new lines at $195 \mathrm{~m} / z$ and $201 \mathrm{~m} / \mathrm{z}$ have evolved with heights of 600 and 700, respectively, the height of the glucose line and that of the isotope line have decreased from 5500 and 5650 to 2300 and 2500 , respectively. The lines at $\mathrm{m} / \mathrm{z} 199.3$ are attributed to impurities. Thus, the mass spectra indicate the conversion of glucose to gluconolactone occurring at the surface of the GOx-immobilized electrode.

\section{$V_{G}$-dependence of glucose oxidation current}

The glucose-gluconolactone conversion concerned here is the result of the oxidation of glucose catalyzed by GOx. Oxidation of glucose results in the transfer of electrons into the electrode with proper enzyme-electrode interfacing. Such interfacing includes redox hydrogels, ${ }^{18}$ nanoparticles ${ }^{6}$ or selected bare electrodes such as pyrolytic graphite and the native surface of silicon wafers. ${ }^{19,20}$ To show the effect of $V_{\mathrm{G}}$ on the glucose oxidation current, cyclic voltammetry was performed using GOx-immobilized electrodes. Fig. 2(a) shows voltammograms 

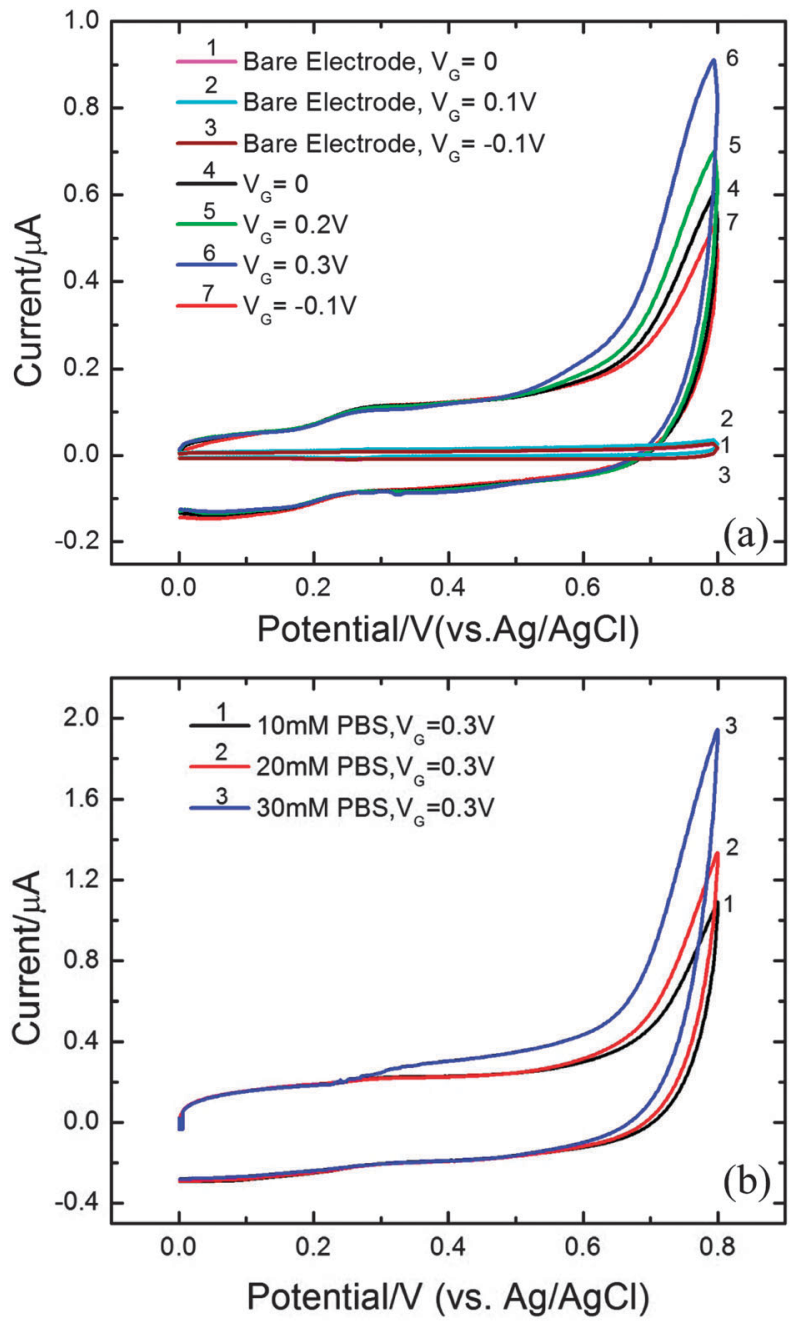

Fig. 2 Electrochemical studies of the oxidation of glucose occurring at GOx immobilized electrodes. (a) The $V_{G}$ dependence of the glucose oxidation current in $10 \mathrm{mM}$ PBS. (b) The effect of ion concentration in the sample on the oxidation current.

(CVs) obtained under different conditions in $10 \mathrm{mM}$ PBS. CV1, $\mathrm{CV} 2$ and CV3 are the results of control experiments, obtained using bare graphite electrodes in $10 \mathrm{mM}$ glucose with $V_{\mathrm{G}}=0 \mathrm{~V}$, $0.1 \mathrm{~V}$ and $\quad 0.1 \mathrm{~V}$, respectively. CV4 was recorded using a GOximmobilized electrode with $V_{\mathrm{G}}=0 \mathrm{~V}$ in the presence of $10 \mathrm{mM}$ glucose. The sharp increase in the anodic current of CV4 indicates the oxidation of glucose catalyzed by GOx. Fig. 2(a) also shows that the anodic currents of GOx-immobilized electrodes (CV5-CV7) in $10 \mathrm{mM}$ glucose depended on both the magnitude and polarity of $V_{\mathrm{G}}$. CV5 and CV6 indicate that the glucose oxidation current could be amplified by increasing $V_{\mathrm{G}}$ in the positive polarity. Detection of glucose on the picomolar level and zepto-molar level has been achieved due to the intrinsic amplification of FEED. ${ }^{8,9}$ CV7 shows that when the polarity of $V_{\mathrm{G}}$ was reversed, the oxidation current became less than that obtained with $V_{\mathrm{G}}=0 \mathrm{~V}$. The increase in the oxidation current due to increasing $V_{\mathrm{G}}$ in the positive polarity as shown by CV5 and CV6 can be attributed to the reduced effective tunnel barrier as depicted in Fig. S1(c), ESI. $\nmid$ The decreased oxidation current as indicated by CV7 is consistent with an increased barrier due to the negative polarity of $V_{\mathrm{G}} \cdot{ }^{15}$ Therefore, the results presented in Fig. 2(a) qualitatively support the scenario depicted in Fig. S1(b) and (c), ESI.†

\section{Dependence of glucose oxidation current on ion concentration}

To provide additional evidence for the mechanism depicted in Fig. S1(b) and (c), ESI, $\uparrow$ the dependence of the glucose oxidation current on the ion concentration of PBS was investigated. Fig. 2(b) shows CVs recorded using a GOx-immobilized electrode in the presence of $10 \mathrm{mM}$ glucose and different PBS ion concentrations. The CVs, obtained with $V_{\mathrm{G}}=0.3 \mathrm{~V}$, show that the glucose oxidation current increased as the PBS concentration was increased. This phenomenon is consistent with the scenario that the ions induced at the electrode-solution interface set up an electric field which modulates the height of the tunnel barrier. As the ion concentration of the PBS is increased, more charges are induced and therefore the field becomes stronger, resulting in further lowered tunnel barrier.

\section{Detection on the zepto-molar level}

Previously, detection of glucose using FEED has been demonstrated on the zM level. ${ }^{9}$ In order to show that the ultrasensitive detection due to the intrinsic signal amplification (see Fig. 2(a)) is not limited to the detection of glucose, we show here the zM-level detection of $\mathrm{H}_{2} \mathrm{O}_{2}$ using electrodes immobilized with microperoxidase-11 (MP-11), a redox biomolecule that catalyzes the reduction of $\mathrm{H}_{2} \mathrm{O}_{2}$ to water. ${ }^{21}$ Fig. 3 shows the zM-level $\mathrm{H}_{2} \mathrm{O}_{2}$ calibration curves, obtained using $V_{\mathrm{G}}=0 \mathrm{~V}$ and $V_{\mathrm{G}}=0.3 \mathrm{~V}$. Each data point on the curves was obtained by measuring the reduction peak current of $\mathrm{MP}-11$ on the $\mathrm{CV}$ obtained at a particular $\mathrm{H}_{2} \mathrm{O}_{2}$ concentration (the CVs are not shown). When $V_{\mathrm{G}}=0 \mathrm{~V}$, the electrode showed no response to changing $\mathrm{H}_{2} \mathrm{O}_{2}$ concentration. With $V_{\mathrm{G}}=0.3 \mathrm{~V}$, the electrode responded to

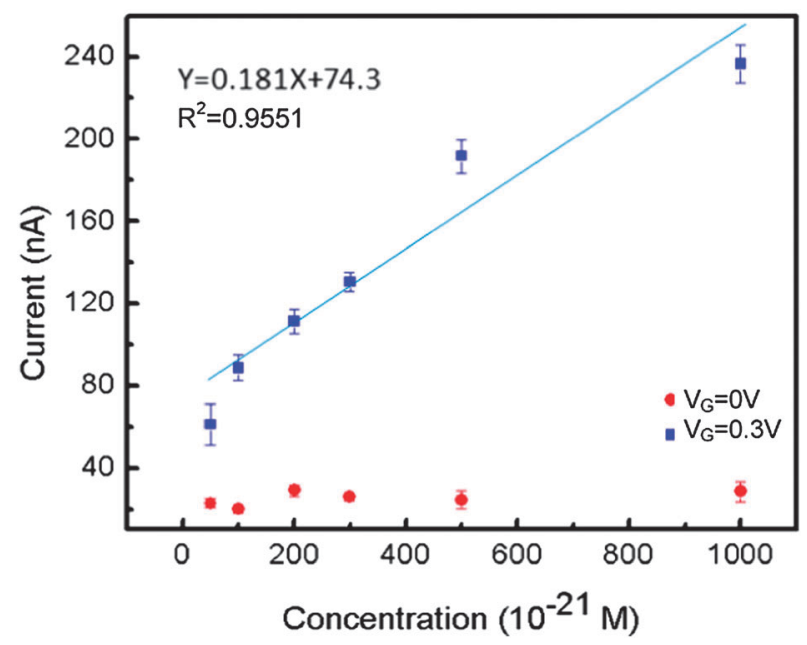

Fig. 3 Zepto molar level calibration curves of $\mathrm{H}_{2} \mathrm{O}_{2}$ obtained with $V_{G}=0 \mathrm{~V}$ and $0.3 \mathrm{~V}$. The straight line is the regression line for the data points, having a correlation coefficient of 0.9551 . 
increasing $\mathrm{H}_{2} \mathrm{O}_{2}$ concentration by increasing the signal current with a detection limit of $42.7 \mathrm{zM}$ (signal/noise = 3). The sensitivity is $1.81 \times 10^{20} \mathrm{nA} \mathrm{M}^{-1}$. The straight line is the regression line for the data points, having a correlation coefficient of 0.9551 .

\section{$V_{\mathrm{G}}$-dependent depletion of glucose}

To provide evidence for the mechanism of FEED from a different perspective, we have measured the change in the glucose concentration of glucose samples (without deaeration) for different values of $V_{\mathrm{G}}$. Fig. 4 shows a set of time traces of glucose concentration. To obtain the traces, glucose samples were prepared by immersing GOx-immobilized electrodes in $2 \mathrm{~mL}$ of $12.7 \mathrm{mM}$ glucose solution and setting the cell potential at $0.8 \mathrm{~V}$ for a specific period of time at a specific value of $V_{\mathrm{G}}$. The glucose concentration of the samples was measured at the end of the time period using a commercial home-blood glucose meter and test strips. Several measurements were made at different times within a total period of $700 \mathrm{~s}$. The control trace was obtained using a blank graphite electrode. Error bars were constructed with three measurements, using three electrodes under identical conditions. Trace 1 shows that, without applying $V_{\mathrm{G}}$, the glucose concentration underwent a continuous decrease during the $700 \mathrm{~s}$ period with a total decrease of $13.7 \%$ of the initial concentration. Trace 1 can be used as a reference for showing the effect of $V_{\mathrm{G}}$.

Trace 2 through Trace 5 show the effect of increasing $V_{\mathrm{G}}$ on the change of glucose concentration. As $V_{\mathrm{G}}$ became more positive, the initial depletion of glucose in the sample solution occurred at progressively faster rates as reflected in these traces. After $240 \mathrm{~s}$, the rates became approximately the same. Trace 5 shows that, with $V_{\mathrm{G}}=0.8 \mathrm{~V}$, the glucose concentration underwent a continuous decrease during the $700 \mathrm{~s}$ period with a total decrease of $33.3 \%$ of the initial concentration. On the contrary, Trace 6 and Trace 7 show that when $V_{\mathrm{G}}$ became more negative, the depletion of glucose became progressively slower than that indicated by Trace 1. The results presented in Fig. 4 are consistent with the scenario depicted in Fig. S1(b) and (c), ESI. $†$

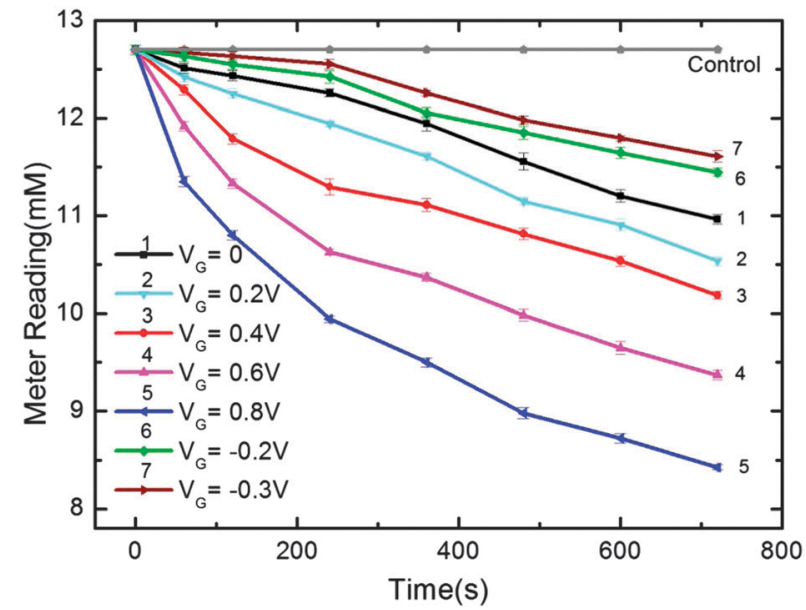

Fig. 4 The effect of $V_{\mathrm{G}}$ on the time dependent depletion of glucose in the sample.
Positive $V_{\mathrm{G}}$ lowers the tunnel barrier so that the conversion of glucose speeds up whereas negative $V_{\mathrm{G}}$ increases the barrier to slow down the conversion. With reference to the results of mass spectrometry shown in Fig. 1, the faster rate of the depletion of glucose caused by increasing $V_{\mathrm{G}}$ was due to the faster conversion of glucose to gluconolactone catalyzed by GOx as the result of enhanced interfacial electron transfer. Further, the $V_{\mathrm{G}}$-dependence of the depletion of glucose shows that the rate of the glucose-gluconolactone conversion can be controlled by $V_{\mathrm{G}}$.

\section{$V_{\mathrm{G}}$-dependence of the kinetics of the} glucose-gluconolactone conversion

The results of Section 3.5 suggest that the kinetics of the enzymatic reaction responsible for the glucose-gluconolactone conversion can be controlled by $V_{\mathrm{G}}$. FEED allowed us to gain insight into the observed effect of $V_{\mathrm{G}}$ on the glucosegluconolactone conversion (see Fig. 4) by providing means to control the kinetic properties of the conversion reaction. Fig. 5(a) shows the $V_{\mathrm{G}}$-dependent glucose calibration curves obtained by recording CVs using identical GOx-immobilized electrodes in solutions of different glucose concentrations and measuring the
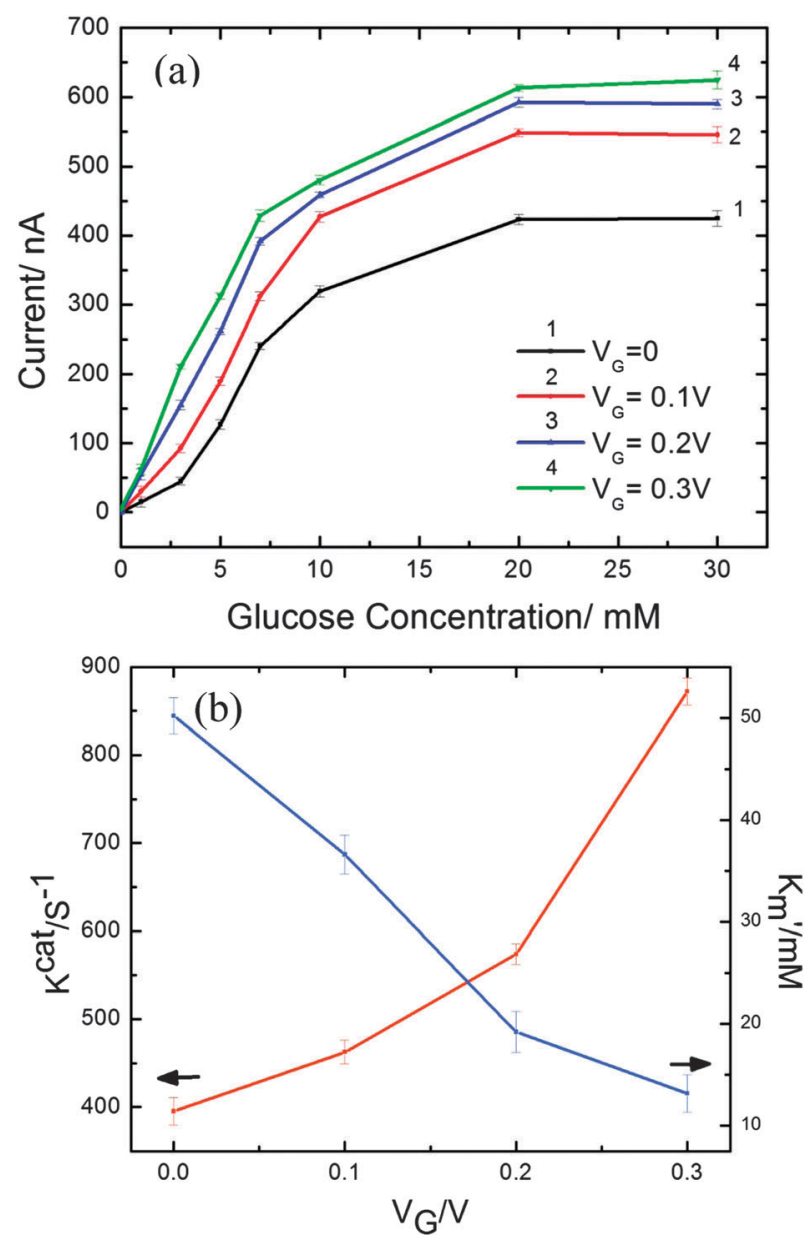

Fig. 5 Controlling the kinetics of the glucose gluconolactone conversion using $V_{\mathrm{G}}$. (a) $V_{\mathrm{G}}$ dependent glucose calibration curves. (b) The $V_{\mathrm{G}}$ dependent $k_{\text {cat }}$ and $K_{\mathrm{m}}{ }^{\prime}$. 
anodic/oxidation current on the CVs at $0.8 \mathrm{~V}$. This procedure was repeated for different values of $V_{\mathrm{G}}$. The effect of $V_{\mathrm{G}}$ on the CVs at several characteristic glucose concentrations is shown in the ESI. $†$

The kinetics of the oxidation of glucose occurring at GOximmobilized electrodes can be characterized using two parameters, namely, $k_{\text {cat }}$ and $K_{\mathrm{m}}{ }^{\prime}$. The turnover rate constant, $k_{\text {cat }}$ is the maximum number of glucose molecules that can be converted to gluconolactone per second per active site and $K_{\mathrm{m}}{ }^{\prime}$ is the apparent Michaelis constant ${ }^{22}$ obtained with GOx immobilized on electrodes. $K_{\mathrm{m}}{ }^{\prime}$ is the glucose concentration required for the speed of the conversion to reach half of its maximum speed. ${ }^{23}$ A low $K_{\mathrm{m}}{ }^{\prime}$ indicates high catalytic efficiency of GOx. ${ }^{23}$ The Lineweaver-Burk (LB) equation relates $I_{\text {ox }}, k_{\text {cat }}$ and $K_{\mathrm{m}}{ }^{\prime}$ as, ${ }^{24}$

$$
1 / I_{\mathrm{ox}}=\left\{K_{\mathrm{m}}{ }^{\prime} /\left(n F A k_{\text {cat }} \Gamma[\text { glucose }]\right)+1 /\left(n F A k_{\text {cat }} \Gamma\right)\right\}
$$

where, $I_{\mathrm{ox}}$ is the oxidation current measured at $V_{\text {cell }}=0.8 \mathrm{~V}$ (the current in Fig. 5(a)), $n$ the number of electrons exchanged, $F$ the Faraday constant, $A$ the electrode surface area, and $\Gamma$ the electrode's surface coverage by GOx. The equation shows that $1 / I_{\text {ox }}$ has a linear dependence on $1 /$ [glucose]. Therefore, $k_{\text {cat }}$ and $K_{\mathrm{m}}{ }^{\prime}$ can be determined from the slope and the vertical intercept of the LB plot. The number of electrons exchanged, $n$, between GOx and the electrode can be estimated using the Laviron equation, $I_{\mathrm{ox}}=(n F Q \nu) /(4 R T)$, where $\nu$ is the scan rate, $Q$ is the charge of an electron, and $F$ is the Faraday constant. Therefore, at room temperature with $v=30 \mathrm{v} \mathrm{s}^{-1}$ and $n=2.2, \Gamma$ can be estimated using the equation $\Gamma=\left(4 I_{p, r} R T\right) /\left(n^{2} F^{2} A v\right)$. Using $1 \mathrm{~mm}^{2}$ for the electrode surface area $A, \Gamma$ was estimated to be $1.125 \times 10^{-11} \mathrm{~mol} \mathrm{~cm}^{-2}$. Since the size of GOx is approximately $6 \mathrm{~nm} \times 5.2 \mathrm{~nm} \times 7.7 \mathrm{~nm}$, the value of $\Gamma$ corresponds to a monolayer.

The dependence of $k_{\text {cat }}$ and $K_{\mathrm{m}}{ }^{\prime}$ on $V_{\mathrm{G}}$ was evaluated by applying the LB technique to the $V_{\mathrm{G}}$-dependent calibration curves shown in Fig. 5(a) and is plotted in Fig. 5(b). Fig. 5(b) shows that $k_{\text {cat }}$ increased and $K_{\mathrm{m}}{ }^{\prime}$ decreased as $V_{\mathrm{G}}$ was increased. In general, a bio-catalytic reaction is described as $\mathrm{E}+\mathrm{S} \underset{k_{1}}{\stackrel{k_{1}}{\rightleftharpoons}} \mathrm{ES} \stackrel{k_{2}}{\longrightarrow} \mathrm{E}+\mathrm{P}$, where $\mathrm{E}$ is the enzyme, $\mathrm{S}$ the substrate, ES the enzyme-substrate complex (bound state) and $\mathrm{P}$ the product. The second step is the catalytic step, in which the product is formed and the enzyme returns to its original state. Fig. 5(b) shows that increasing $V_{\mathrm{G}}$ resulted in a faster conversion process from glucose to gluconolactone catalyzed by a GOx molecule. This is because electron transfer is required to initiate the second step. Therefore, enhanced $k_{\text {et }}$ at the GOxelectrode interface due to the field resulted in a higher $k_{\text {cat }}\left(=k_{2}\right)$ so that more gluconolactone molecules were produced and GOx molecules returned to their unbound state more quickly to be available for the next conversion process. Therefore, the required glucose concentration for the system to reach half of its maximum velocity $\left(K_{\mathrm{m}}{ }^{\prime}\right)$ became lowered.

In Fig. 5(b), $k_{\text {cat }}$ shows a faster increase starting at $V_{\mathrm{G}}=0.2 \mathrm{~V}$, indicating a nonlinear dependence on $V_{\mathrm{G}}$. This nonlinearity is likely to be the result of the exponential dependence of $k_{\text {et }}$ on $\beta$ as shown in Section 1.1. In fact, the nonlinear dependence of glucose depletion on $V_{\mathrm{G}}$ shown in Fig. 4 could be a result of the observed nonlinear dependence of $k_{\text {cat }}$ on $V_{\mathrm{G}}$. The voltageenhanced reaction kinetics is qualitatively supported by the behavior of $K_{\mathrm{m}}{ }^{\prime}$ as shown in Fig. 5(b), where $K_{\mathrm{m}}{ }^{\prime}$ decreases as $V_{\mathrm{G}}$ is increased, indicating higher bio-catalytic efficiency.

The results presented in this section and Section 3.5 indicate that the kinetics of the glucose-gluconolactone conversion can be controlled using an external voltage. This capability has important implications. In cellular metabolism of glucose, both aerobic and anaerobic respiration pathways involve transfer of electrons by means of redox enzymes such as the different kinds of dehydrogenases. ${ }^{25}$ Glucose is eventually converted to $\mathrm{CO}_{2}$ or ethanol. The present work indicates a possible approach to control cellular metabolism of glucose. Since the cytoplasm and the mitochondrion contain ions, $V_{\mathrm{G}}$ re-arranges the ions around redox enzymes involved in the glucose metabolism to set up electric fields, which facilitate the electron transfer and therefore speeds up the metabolic process. The enzymes do not need to be in contact with the electrode. An envisioned hypothetical example is the reduction of $\mathrm{NAD}^{+}$by glyceraldehyde3-phosphate dehydrogenase in glycolysis or the passage of electrons from $\mathrm{NADH}$ to $\mathrm{NADH}$ dehydrogenase in the electron transport chain and the subsequent traversing through the ionsulfur centers in the enzyme. Also, the results of this section suggest that the kinetics of a general redox enzymatic reaction occurring at an electrode can be controlled by controlling interfacial electron transfer using $V_{\mathrm{G}}$. Presently, external control of electron transfer and reaction kinetics in biological systems is performed mainly optically. ${ }^{26}$ It was proposed that, by controlling the rate of electron transfer in metabolic systems using certain chemical reactions, metabolism can be regulated to avoid detrimental oxidative stress effects. ${ }^{27}$ It was predicted that the activation energy that determines electron transfer efficiency across a biological membrane can be controlled using electrostatic means. ${ }^{28}$ Until now, the only demonstrated electrostatic control of electron transfer in proteins was the change in the interfacial current as the result of the reorientation of cytochrome $\mathrm{c}$ on the working electrode caused by $V_{\text {cell. }}{ }^{29-31}$ The present work indicates the feasibility of controlling electron transfer in biological systems using electrostatic means.

\section{Conclusion}

This article presents a series of experiments, whose results, while providing evidence for the proposed mechanism of FEED, demonstrate the feasibility of using an external voltage to control the kinetics of the glucose-gluconolactone conversion. In particular, the scenario that the $V_{\mathrm{G}}$-induced ions set up an electric field at the solution-electrode interface is supported by the observed dependence of the glucose oxidation current on $V_{\mathrm{G}}$ and the ion concentration of the sample solution. Also, the $V_{\mathrm{G}}$-dependent depletion of glucose in the sample solution provides additional evidence for the proposed mechanism. Further, the $V_{\mathrm{G}}$-controlled depletion of glucose provides evidence that the glucose-gluconolactone conversion can be controlled 
using an external voltage. The demonstrated dependence of the kinetics of the glucose-gluconolactone conversion on $V_{\mathrm{G}}$ indicates the feasibility of controlling glucose metabolism in microorganisms using electrodes and an external voltage. The mechanism of FEED suggests that the technique can be extended to other conversion processes catalyzed by a redox enzyme such as glucose metabolism in microorganisms. In the broader sense, the results reported in this work can be used to improve the performance of enzyme-based and microbial biosensors.

\section{Acknowledgements}

We thank the support of Cleveland State University.

\section{References}

1 J. N. Onuchic, C. Kobayash, O. Miyashita, P. Jennings and K. K. Baldridge, Philos. Trans. R. Soc. London, Ser. B, 2006, 361, 1439-1443.

2 J. M. Nocek, J. S. Zhou, S. D. Forest, S. Priyadarshy, D. N. Beratan, J. N. Onuchic and B. M. Hoffman, Chem. Rev., 1996, 96, 2459-2489.

3 S. K. Chaudhuri and D. R. Lovely, Nat. Biotechnol., 2003, 21, 1229-1232.

4 C. Dellomonaco, J. M. Clomburg, E. N. Miller and R. Gonzalez, Nature, 2011, 476, 355-361.

5 A. J. Wargacki, E. Leonard, M. N. Win, D. D. Regitsky, C. N. Santos, P. B. Kim, S. R. Cooper, R. M. Raisner, A. Herman, A. B. Sivitz, A. Lakshmanaswamy, Y. Kashiyama, D. Baker and Y. Yoshikuni, Science, 2012, 335, 308-313.

6 Y. Xiao, F. Patolsky, E. Katz, J. F. Hainfeld and I. Willner, Science, 2003, 299, 1877-1881.

7 T. S. Wong and U. Schwaneberg, Curr. Opin. Biotechnol., 2003, 14, 590-596.

8 Y. Choi and S.-T. Yau, Anal. Chem., 2009, 81, 7123-7126.

9 Y. Choi and S.-T. Yau, Biosens. Bioelectron., 2011, 26, 3386-3390.

10 S. J. Tans, A. R. M. Verschueren and C. Dekker, Nature, 1998, 393, 49-52.
11 S.-T. Yau and G. Qian, Appl. Phys. Lett., 2005, 86, 103508.

12 Y. Choi and S.-T. Yau, AIP Adv., 2011, 1, 042175.

13 Biotechnological innovations in chemical synthesis, ed. M. C. E. v. Dam-Mieas and C. K. Leach, Butterworth-Heinemann, Oxford, 1997.

14 R. A. Marcus and N. Sutin, Biochim. Biophys. Acta, 1985, 811, 265-322.

15 J. D. Jackson, Classical Electrodynamics, John Wiley \& Sons, Inc., Danvers, 1998.

16 S. J. Tans, A. R. M. Verschueren and C. Dekker, Nature, 1998, 393, 49-52.

17 S. J. Tans and C. Dekker, Nature, 2000, 404, 834-835.

18 A. Heller and B. Feldman, Chem. Rev., 2008, 108, 2482-2505.

19 G. Wang, N. M. Thai and S.-T. Yau, Electrochem. Commun., 2006, 8, 987-992.

20 G. Wang and S.-T. Yau, Biosens. Bioelectron., 2007, 22, 2158-2164.

21 P. Turano and Y. Lu, in Handbook on Metalloproteins, ed. I. Bertini, A. Sigel and H. Sigel, Marcel Dekker, New York, 2001.

22 R. A. Kamin and G. S. Wilson, Anal. Chem., 1982, 52, 1198-1205.

23 D. J. Voet and J. G. Voet, Biochemistry, Wiley, New York, 1995.

24 A. S. Kumar and J.-M. Zen, Electroanalysis, 2002, 14, 671-678.

25 C. K. Mathews, K. E. vanHold and K. G. Ahern, Biochemistry, Addison Wesley Longman, San Francisco, 1999.

26 B. A. Heller, D. Holten and C. Kirmaier, Science, 1995, 269, 940-945.

27 V. L. Davidson, Biochemistry, 2002, 41, 14633-14636.

28 A. Warshel and D. W. Schlosser, Proc. Natl. Acad. Sci. U. S. A., 1981, 78, 5564-5568.

29 J. L. Willit and E. F. Bowden, J. Electroanal. Chem., 1987, 221, 265-274.

30 E. Strauss, B. Thomas and S.-T. Yau, Langmuir, 2004, 20, 8768-8772.

31 A. Kranich, H. K. Ly, P. Hildebrandt and D. H. Murgida, J. Am. Chem. Soc., 2008, 130, 9844-9848. 